\title{
Numerical Evaluation of Heat Transfer and Entropy Generation of Helical Tubes with Various Cross-sections under Constant Heat Flux Condition
}

\author{
Jundika Candra Kurnia 1, *, Agus Pulung Sasmito \\ ${ }^{1}$ Mechanical Engineering Department, Universiti Teknologi PETRONAS, 32610 Bandar Seri \\ Iskandar, Perak Darul Ridzuan, Malaysia. \\ ${ }^{2}$ Department of Mining and Materials Engineering, McGill University, 3450 University Street, Frank \\ Dawson Adams Bldg, Montreal Quebec H3A 2A7 Canada.
}

\begin{abstract}
The presence of curvature-induced secondary flow in helical pipe which create complex transport phenomena and higher transfer rate has attracted significant attention from both academic and industry. Flow behavior and transport processes in helical tube have been intensively investigated. Nevertheless, most studies were focused on the performance based on first law of thermodynamics with limited studies concerning the performance based on second law of thermodynamics. The objective of this study is to investigate the heat transfer performance of helical tube according to both first and second law. The heat transfer rate and entropy generation of helical tubes with various cross-sections, i.e. circular, ellipse and square, subjected to constant wall heat flux conditions are numerically evaluated by utilizing computational fluid dynamics (CFD) approach. Their performances are compared to those of straight tube with identical cross-section. The results indicate that helical tube provides higher heat transfer at the cost of higher pressure. Moreover, it was found that entropy generation in helical tubes is considerably lower as compared to that in straight tube. Among the studied cross-sections, square has the highest heat transfer albeit having the highest pressure drop and entropy generation for both straight and helical tubes.
\end{abstract}

\section{Introduction}

The complex transport processes occur in helical tube due to the presence of curvatureinduced secondary flow has attracted considerable attention from researcher worldwide. Initiated by the pioneer work of Dean $[1,2]$ using Toroidal tube, a string of studies on helical tube have been carried out. Characteristic of secondary flow in helical tube and other coiled tubes are primarily dictated by Dean Number, a dimensionless parameter which is defined as the ratio of the viscous force acting on a fluid flowing in a curved pipe to the centrifugal

\footnotetext{
*Corresponding author: jundika.kurnia@utp.edu.my; kurnia.jc@gmail.com
} 
force. Secondary flow was observed when the Dean number exceeds critical value and it can appears as one pair or two pairs depending on the Dean number $[3,4]$.

Subsequent studies revealed that helical tube provides higher heat transfer at smaller footprints as compared to straight tube. As such, many industrial processes have adopted helical tubes to achieve higher performance. The most common industrial applications that utilize helical tubes are heat exchanger and chemical reactors. Driving by the need to continuously improve and intensify heat transfer process, a vast number of studies on the heat transfer performance of helical tubes have been conducted and reported.

Kurnia et al numerically investigate several key parameters affecting the heat transfer performance of coiled tubes including coil geometries [5], cross-sections [6], Reynolds and Prandtl number [7,8], non-Newtonian fluid [9] and addition of nano particle [10]. They evaluated the performance by utilizing figure of merit, a ratio heat transfer per unit pumping power required. Kong et al [11] investigated the heat transfer performance of microencapsulated phase change materials (MEPCM) in a heated helically coiled tube. They found that MEPCM marginally enhance heat transfer within the helical tube. The reason for this minimum enhancement is high viscosity and low latent heat of fusion of the PCM.

Wu et al [12] and Aly [13] evaluated heat transfer performance of nanofluid in annular helically coiled tube. They found that addition nanoparticle to the base fluid does increase heat transfer in the annular helical tube. Tohidi [14] introduced chaotic advection to increase heat transfer in helically coiled tubes and found that an increased in heat transfer up to $26 \%$ can be achieved with up to $8 \%$ changes in pressure drop. The effect or curvature radius on the heat transfer performance of laminar flow of nanofluid in helically coiled tube was reported by Mirfendereski et al [15]. The results indicated that larger curvature offers higher heat transfer rate enhancement. Alimoradi and Veysi [16] experimentally and numerically investigate heat transfer of helical tube in shell and tube configuration. They found that Nusselt number is affected by tube and negligibly influenced by the coil pitch. To enhance the heat transfer performance of helical tube, Zachar [17] introduced helical corrugation to the tube. They achieved higher heat transfer rate at the cost of higher pressure drop.

In these studies, the heat transfer performance of helical tube was evaluated based on the first law of thermodynamics. While useful in determining the performance, it cannot identify the root cause of inefficiency in a system.

Recently, evaluation based second law thermodynamics is increasingly adopted as it allows for identification on the cause of inefficiency in the system [18]. Recently, we carry out study on heat transfer performance and entropy generation of laminar flow in helical tubes with various cross-sections subjected to constant wall temperature. The results indicated that albeit inducing higher pressure drop, helical coil offers higher heat transfer and lower entropy generation. Here, we continue our study by evaluating the heat transfer performance and entropy generation helical non-circular tube with various cross-sections subjected to constant wall heat flux. For this purpose, a computational fluid dynamics approach which is coupled with entropy generation analysis is adopted. The performance of each configuration will be discussed based on the numerical simulation findings.

\section{Mathematical modelling}

To evaluate the laminar heat transfer in helical tube with various cross-section, a three dimensional CFD model is developed and validated. The model takes into account the conservation of mass, momentum and energy for an incompressible laminar Newtonian fluid. The heat transfer fluid considered in this study is air. Figure 1 present the schematic diagram of the model which is similar to those in our previous study [19]. The physical characteristics of the studied geometries are summarized in Table 1. To properly compare heat transfer of the studied geometries, the total length of all tubes is similar. 
a)
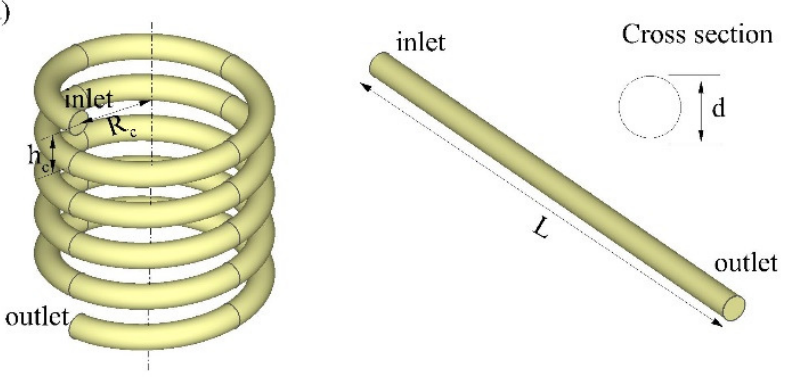

b)
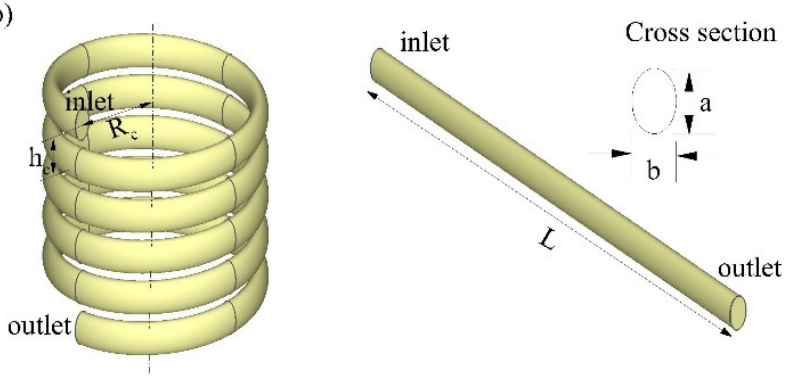

c)
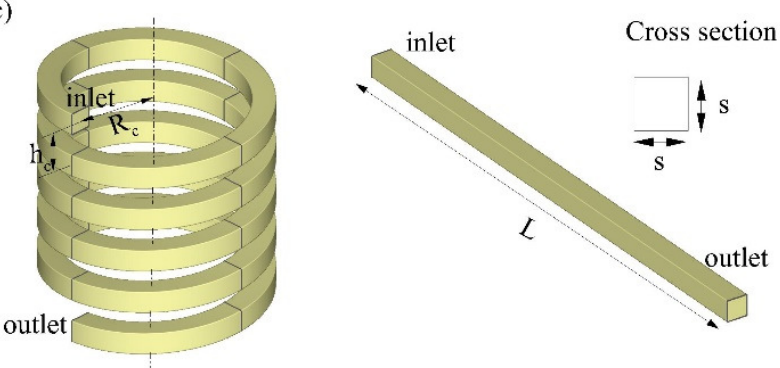

Fig. 1. Schematic diagram of the experiment setup.

Table 1. Geometrical parameters of studied straight and helical tubes.

\begin{tabular}{|c|c|c|}
\hline Parameters & Value & Unit \\
\hline $\mathrm{a}$ & $7.98 \times 10^{-3}$ & $\mathrm{~m}$ \\
\hline $\mathrm{b}$ & $1.60 \times 10^{-2}$ & $\mathrm{~m}$ \\
\hline $\mathrm{d}$ & $1.13 \times 10^{-2}$ & $\mathrm{~m}$ \\
\hline $\mathrm{h}_{\mathrm{c}}$ & $1.13 \times 10^{-2}$ & $\mathrm{~m}$ \\
\hline $\mathrm{L}$ & 1.26 & $\mathrm{~m}$ \\
\hline $\mathrm{R}_{\mathrm{c}}$ & $4.00 \times 10^{-2}$ & $\mathrm{~m}$ \\
\hline $\mathrm{s}$ & $1.00 \times 10^{-2}$ & $\mathrm{~m}$ \\
\hline
\end{tabular}




\subsection{Governing equations}

The conservation equation of mass, momentum and energy for the flow inside the tubes can be expressed as,

$$
\begin{aligned}
& \nabla \cdot \rho \mathbf{u}=0 \\
& \nabla \cdot(\rho \mathbf{u u})=-\nabla p \mathbf{I}+\nabla \cdot\left[\mu\left(\nabla \mathbf{u}+(\nabla \mathbf{u})^{T}\right)\right]+\rho \mathbf{g}, \\
& \rho c_{p} \mathbf{u} \cdot \nabla T=k \nabla^{2} T
\end{aligned}
$$

where $\rho$ is the fluid density, $\mathbf{u}$ is the velocity, $p$ is the pressure, $\mu$ is the dynamic viscosity, $\mathbf{I}$ is identity tensor, $\mathbf{g}$ is gravity acceleration, $c_{p}$ is the specific heat, $k$ is thermal conductivity and $T$ is the temperature.

Similar to our previous study [19], to investigate the entropy generation in the studied geometries, entropy balanced equation for open system is adopted. For continuum framework - the one considered in this study - the second law thermodynamic can be expressed as:

$$
-\nabla \boldsymbol{\sigma}+s_{g}=0
$$

where $\sigma$ and $\mathrm{s}_{\mathrm{g}}$ are the entropy flux and the entropy generation rate per unit volume, respectively.

\subsection{Constitutive relations}

The temperature dependent properties of air are taken into account by adopting polynomial function which is formulated based on the air properties taken from Kays et al [20]. [6]:

The air properties, valid within the range of $298 \mathrm{~K}-398 \mathrm{~K}$, are represented as the followings

$$
\begin{aligned}
& \rho=1.076 \times 10^{-5} T^{2}-1.039 \times 10^{-3} T+3.326, \\
& \mu=5.21 \times 10^{-15} T^{3}-4.077 \times 10^{-11} T^{2}+7.039 \times 10^{-8} T+9.19 \times 10^{-7}, \\
& k=4.084 \times 10^{-10} T^{3}-4.519 \times 10^{-7} T+2.35 \times 10^{-4} T-0.0147, \\
& c_{p}=-4.67 \times 10^{-6} T^{3}+4.837 \times 10^{-3} T^{2}-1.599 T+1175 .
\end{aligned}
$$

In this study, the entropy generation rate per unit volume $\left(\mathrm{s}_{\mathrm{g}}\right)$ is considered to consist of 2 component, i.e. heat transfer component $\left(\mathrm{s}_{\mathrm{h}}\right)$ and viscous dissipation component $\left(\mathrm{s}_{\mu}\right)$ which are given by

$$
s_{h}=\frac{\nabla \cdot(k \nabla T)}{T^{2}} \text { and } s_{\mu}=-\frac{1}{T} \tau: \nabla \mathbf{u},
$$

respectively. The viscous term is given by the second term right hand side of conservation of momentum (equation 2). 
To evaluate the heat transfer performance of the studied geometries, the numerical results will be presented in terms of the mixed mean temperature along the tubes, $\mathrm{T}_{\text {mean }}$, total heat transfer, $\dot{Q}$, and Figure of Merit, FoM. The mixed mean temperature is calculated as

$$
T_{\text {mean }}=\frac{1}{V A_{c}} \int_{A_{c}} \mathrm{u} T d A_{c}
$$

where $\mathrm{V}$ is the mixed mean velocity and $\mathrm{A}_{\mathrm{c}}$ is the cross-section area of the tubes. To calculate the mean velocity, the following formula is used, i.e.

$$
V=\frac{1}{A_{c}} \int_{A_{c}} \mathrm{u} d A_{c} .
$$

Once the mixed mean and mean velocity are obtained, the total heat transfer rate and figure of merit can be calculated as

$$
\begin{aligned}
& \dot{Q}_{\text {total }}=\dot{m} c_{p}\left(T_{\text {mean }, L}-T_{\text {mean }, 0}\right), \\
& F o M=\frac{\dot{Q}_{\text {total }}}{P_{\text {pump }}},
\end{aligned}
$$

where the mass flow rate is given by

$$
\dot{m}=\rho A_{c} U_{i n} .
$$

$T_{\text {mean, } L}$ and $T_{\text {mean, } O}$ are the mixed mean temperature at length $L$ and at the channel inlet, respectively. The required pumping power is calculated from the pressure drop and volume flow rate as follows

$$
P_{p u m p}=\frac{1}{\eta_{p u m p}} \dot{V} \Delta p
$$

with $\eta_{\text {pump }}$ being pump efficiency (assumed to be $70 \%$ ) and $\dot{V}$ is the volumetric flow rate of the heat transfer fluid.

The entropy generation is evaluated in term of global entropy generation which is calculated by integrating the entropy rate generation per unit volume over the entire volume, i.e.

$$
\dot{S}_{g}=\int_{\forall} s_{g} d V
$$

\subsection{Boundary conditions}

Boundary conditions for the fluid flow inside the tube are summarized as follows:

- At the walls: no slip boundary condition, and constant wall heat flux ( $\left.\mathrm{q}_{\text {wall }}\right)$ of $283 \mathrm{~W} \mathrm{~m}^{-}$ 2 are applied at the walls.

- At the inlet: velocity corresponding to Reynolds number of 1000 and constant temperature of $298.15 \mathrm{~K}\left(25^{\circ} \mathrm{C}\right)$ are defined at the inlet. 
- At the outlet: the gauge pressure, $p$, and streamwise temperature gradient, $\mathbf{n} \cdot \nabla T$, are set to zero.

\subsection{Numerics}

The computational domain for helical tubes were created using AutoCAD and then exported to ANSYS pre-processing tools for meshing and labelling. Finite volume solver, ANSYS Fluent, was utilised to solve the governing mathematical model, boundary conditions and constitutive relations comprising five dependent variables $-u, v, w, P$ and $T$. User-defined functions (UDF) were written in $\mathrm{C}$ language to incorporate the thermo-physical properties of the fluid and inclusion of entropy generation analysis into the computational model.

To solve the numerical model, Semi-Implicit Pressure-Linked Equation (SIMPLE) algorithm, second-order upwind discretisation and Algebraic Multi-grid (AMG) method were used. In terms of computational cost, a personal computer with a quad core $2.0 \mathrm{GHz}$ processors and 6 GB of RAM took approximately 5-15 minutes for 100-400 iterations to meet the specified convergence criteria of $10^{-6}$.

To ensure that the numerical results is independent of mesh and obtain optimum balance between accuracy and required computational resources, a mesh independent study was conducted by systematically increase the amount of mesh element until no significant changes on key parameters (temperature and pressure) are observed. The results from the mesh size amounting $4 \times 10^{5}$ to $5 \times 10^{5}$ has marginal difference with those of higher element number. Hence this mesh size was chosen for the rest of simulation to provide optimum balance between accuracy and required computational resources.

\section{Results and discussions}

To ensure the validity of the developed model, model prediction is compared to the empirical correlations for straight and helical tubes. Details model validation can be found in our previous work [19]. In short, the model prediction shows good agreement with the empirical correlation, providing confidence on the capability of the model to properly capture transport processes in the considered cases.

Figure 2 presents the cross-section velocity profile inside the tubes. For helical tubes, secondary flow can be clearly observed at the region near the outer wall. This secondary flow is not observed in straight tube where a fully developed flow with the peak velocity in the middle of the tube can be found. Closer inspection reveals that the peak velocity for straight tube is located slightly below the middle due to the effect of buoyancy. In-line with the cases for constant wall temperature, two pair secondary flow is observed in helical tube with ellipse cross-section while helical tube with square and circular cross-section shows single pair of secondary flow.

Looking towards the temperature distribution inside the tube, as presented in Figure 3, it can be easily notified that secondary flow strongly dictates heat transfer inside the tube. As can be seen, higher temperature gradient is observed near the outer wall for all helical tubes, indicating intense heat transfer between the wall and heat transfer fluid. For straight tubes, the effect of buoyancy is more pronounced for the circular cross-section where the shifting of the cold core is more prominent. 


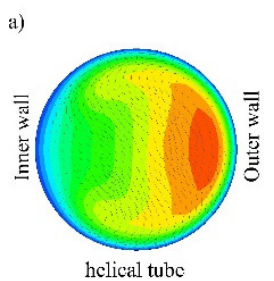

b)

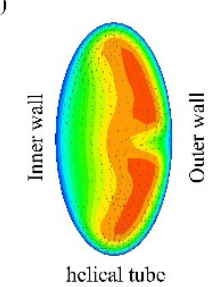

c)

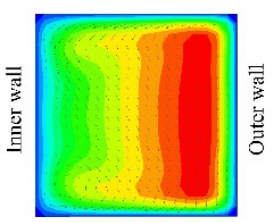

helical tube
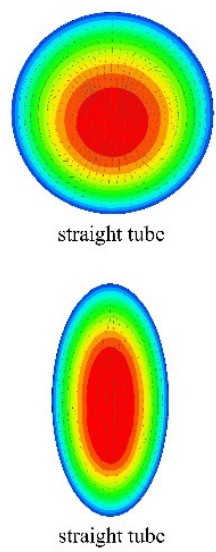

straight tubc

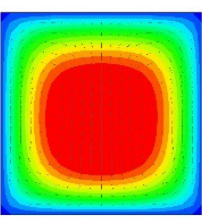

straight tube

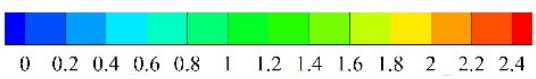

Fig. 2. Axial velocity profile of airflow in helical and straight tubes with a) circular, b)ellipse and c) square cross-sections subjected to constant wall heat flux at $\mathrm{L}=25 \mathrm{~cm}$.

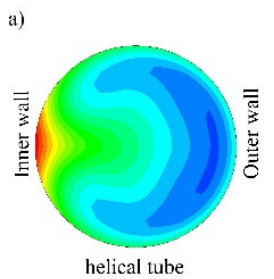

b)

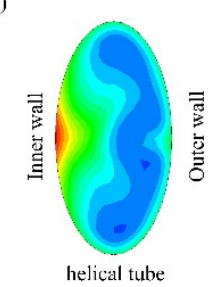

c)

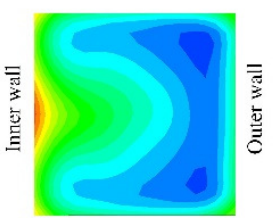

helical tube

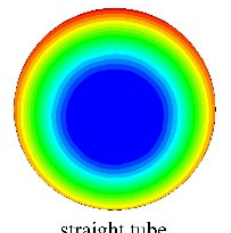

straight tube

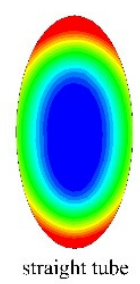

straight tube

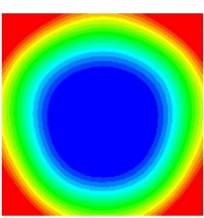

straight tube

$\begin{array}{llllllll}304 & 308 & 312 & 316 & 320 & 324 & 328 & 332\end{array}$

Fig. 3. Temperature distribution in helical and straight tubes with a) circular, b)ellipse and c) square cross-sections subjected to constant wall heat flux at $\mathrm{L}=25 \mathrm{~cm}$. 
Another point of interest in this study is the evaluation of entropy generation in the studied geometry. As presented previously, the entropy generation in thermal system considered in this study comprises 4 main components, i.e. the heat transfer component and fluid flow component. Figure 4 presents local distribution of entropy generations due to heat transfer. As those for constant wall temperature [19], helical tubes generate lesser entropy as compared to the straight counterpart. Higher entropy generation is observed at the area with higher temperature gradient. Nevertheless, we observe different pattern of entropy generation in constant wall heat flux cases as compared to those of constant wall temperature. Here, higher entropy generation for helical tubes occurs at both inner and outer walls whereas for constant wall temperature higher entropy generation is found near the outer wall. For straight tube, higher entropy generation can be found at the corner which in contrast to constant wall temperature cases this location has the lower entropy generation.

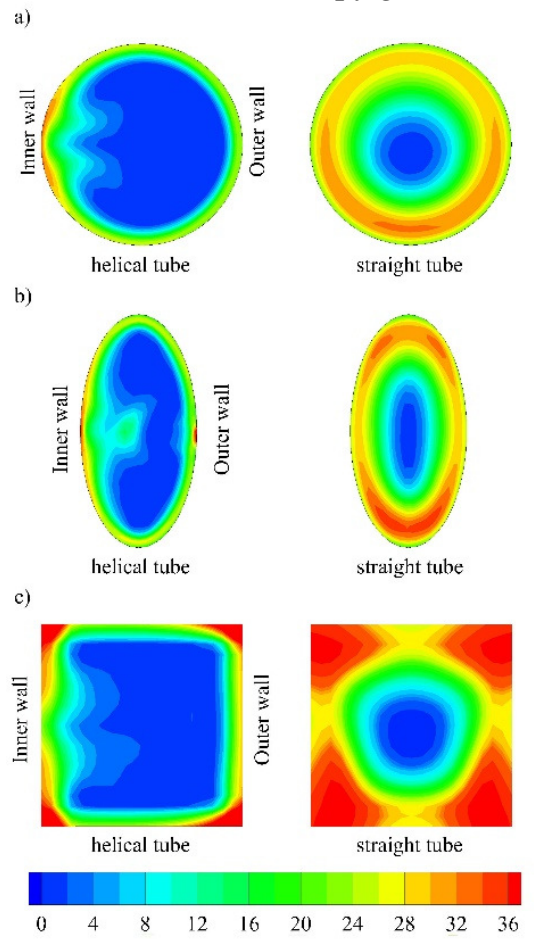

Fig. 4. Local distribution of entropy generation due to heat transfer in helical and straight tubes with a) circular, b)ellipse and c) square cross-sections subjected to constant wall heat flux at $\mathrm{L}=25 \mathrm{~cm}$.

Figure 5 present the local distribution of entropy generation due to viscous dissipation in the studied tubes. In contrast to the entropy generation due to heat transfer, entropy generation due to viscous dissipation is observed higher in helical tubes than that in straight tubes. This is consistent with the finding for constant wall temperature. This is most likely attributed by the presence of the secondary flow inside the helical tubes which not only enhances the heat transfer but also induce higher friction especially at the outer wall region. This certainly reduces the system efficiency and increase entropy generation, as presented in Figure 4. Looking at the scale, however, the contribution of viscous dissipation to entropy generation is significantly lower as compared to heat transfer contribution (almost two order magnitudes lower). As such, the total entropy generation will be primarily dictated by the heat transfer contribution. 


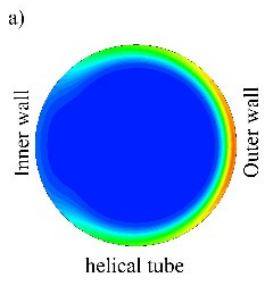

b)

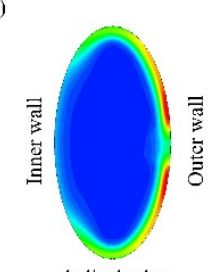

helical tube

c)

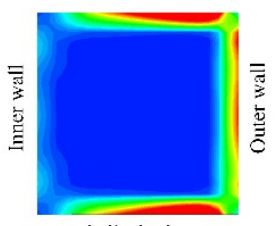

helical tube

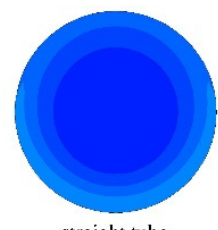

straight tube

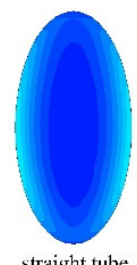

straight tube

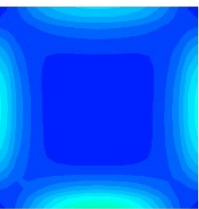

straight tube

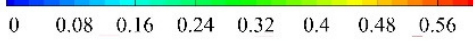

Fig. 5. Local distribution of entropy generation due to heat transfer in helical and straight tubes with a) circular, b)ellipse and c) square cross-sections subjected to constant wall heat flux at $\mathrm{L}=25 \mathrm{~cm}$.

Table 2. Total heat transfer rate, pressure drop and figure of merit for the laminar heat transfer in helical and straight tubes.

\begin{tabular}{|l|l|l|l|}
\hline Geometry & $\begin{array}{l}\text { Heat transfer } \\
\text { rate }(\mathrm{W})\end{array}$ & $\begin{array}{l}\text { Pressure } \\
\text { drop (Pa) }\end{array}$ & $\begin{array}{l}\text { Figure of } \\
\text { Merit (FoM) }\end{array}$ \\
\hline Helical circle & 12.60 & 28.60 & 2234.33 \\
\hline Helical ellipse & 13.27 & 31.58 & 1957.16 \\
\hline Helical square & 13.94 & 36.70 & 1708.92 \\
\hline Straight circle & 11.17 & 12.91 & 4388.09 \\
\hline Straight ellipse & 12.24 & 16.73 & 3410.52 \\
\hline Straight square & 12.60 & 16.69 & 3396.68 \\
\hline
\end{tabular}

A more quantitative comparison between studied geometries can be found in Figure 6 which presents mixed mean temperature, Nusselt number and entropy generation along the tubes as well as tables 2 and 3 which summarizes key performance parameters (heat transfer, pressure drop, FoM and global entropy generation). Overall, helical tubes offer higher heat transfer, indicated by higher mixed mean temperature and higher $\mathrm{Nu}$. Helical tubes also produce lower entropy generation along the tube as compared to the straight counterpart.

Among the studied cross-sections for helical tubes, although circular cross section has higher mean temperature, square cross-section yields higher $\mathrm{Nu}$, indicating better heat transfer rate along the tube. For straight tube, circular cross-section has higher mean and $\mathrm{Nu}$. 
On entropy generation, square cross-section has the highest entropy generation for both helical and straight tubes. For straight tube, however, the generation is closely followed by elliptic cross-section.
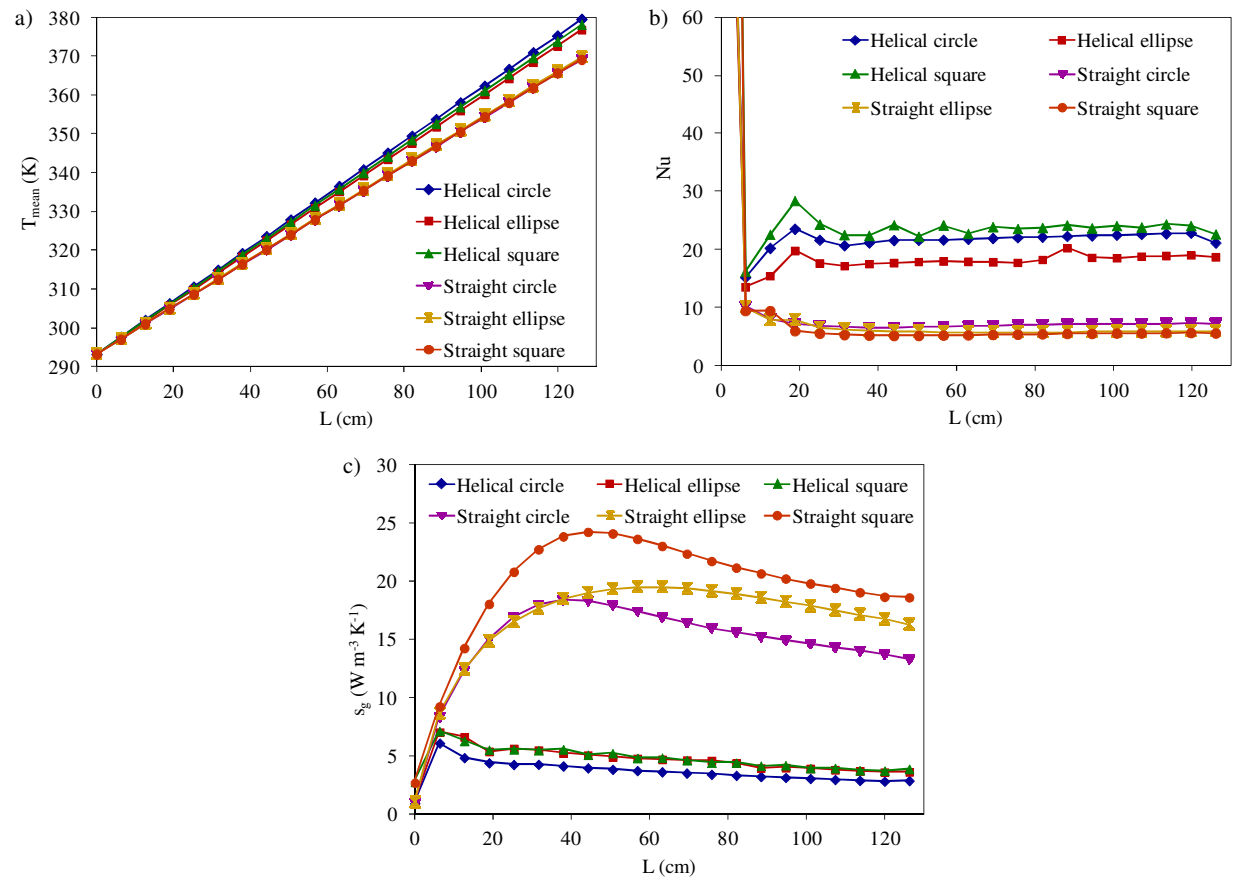

Fig. 6. a) Mixed mean temperature b) Nusselt number and c) Local entropy generation (sg) along the tube length.

Overall, the total heat transfer rate for helical tube is higher as compared to that of straight tube which is attributed to the presence of secondary flow in helical tubes. This higher heat transfer come with penalty of significant increase in pumping power. The pumping power required for helical tubes is almost double of that for straight tube. As a result, FoM for helical tubes is lower albeit their higher heat transfer rate.

Table 3. Global entropy generation inside tube with constant wall temperature.

\begin{tabular}{|l|l|l|l|}
\hline \multirow{2}{*}{ Geometry } & \multicolumn{3}{|l|}{ Global entropy generation $\left(\times 10^{-4} \mathrm{~W} \mathrm{~K}^{-1}\right)$} \\
\cline { 2 - 4 } & $\begin{array}{l}\text { Heat } \\
\text { transfer }\end{array}$ & Viscous & Total \\
\hline Helical circle & 7.79 & 0.10 & 7.89 \\
\hline Helical ellipse & 8.96 & 0.12 & 9.08 \\
\hline Helical square & 10.04 & 0.16 & 10.19 \\
\hline Straight circle & 23.08 & 0.05 & 23.13 \\
\hline Straight ellipse & 25.51 & 0.07 & 25.58 \\
\hline Straight square & 31.41 & 0.07 & 31.48 \\
\hline
\end{tabular}


Looking towards the global entropy generation within the tube, it is found that entropy generation for straight tube is more than twice of those of helical tubes, highlighting the potential of helical tubes. As explained before, the contribution of viscous dissipation on entropy generations is marginal as compared to the contribution of heat transfer.

\section{Conclusions}

Investigation on a laminar heat transfer inside helical tubes with various cross-sections subjected to constant wall heat flux has been carried out. A validated three-dimensional computational fluid dynamics (CFD) model was utilized to evaluate the flow characteristic, heat transfer rate and entropy generation. The investigated cross-sections are circle, ellipse and square. The performance of helical tubes is compared to those of straight tube with similar cross-section.

The result suggested that higher heat transfer is achieved by using helical tubes despite higher pressure drop imposed. It should be noted that entropy generation in helical tubes is considerably lower as compared to that in straight tube. Among the studied cross-sections, square has the highest heat transfer albeit having the highest pressure drop and entropy generation for both straight and helical tubes.

Further studies are currently underway to investigate other parameters to obtain the comprehensive information on optimum operating and geometric condition for helical tubes.

\section{References}

1. W.R. Dean. Philos Mag 4, 208-223 (1927)

2. W.R. Dean, Philos Mag 5, 673-695 (1928)

3. J.H. Masliyah. J Fluid Mech 99, 469-479 (1980)

4. S.C.R. Dennis, M. Ng. Q J Mechanics Appl Math 35, 305-324(1982)

5. J.C. Kurnia, A.P. Sasmito, A.S. Mujumdar. Heat Transfer Eng 37, 1341-1356 (2016)

6. A.P. Sasmito, J.C. Kurnia, W. Wang, S.V. Jangam, A.S. Mujumdar. Int J Heat Mass Tran 55, 5882-5890 (2012)

7. J.C. Kurnia, A.P. Sasmito, A.S. Mujumdar. Therm Sci 16, 109-118 (2012)

8. J.C. Kurnia, A.P. Sasmito, A.S. Mujumdar. J Zhejiang Univ-Sci A (Appl Phys \& Eng) 12, 63-70 (2011)

9. J.C. Kurnia, A.P. Sasmito, A.S. Mujumdar. Int Commun Heat Mass 57, 100-108 (2014)

10. A.P. Sasmito, J.C. Kurnia, A.S. Mujumdar. Nanoscale Res Lett 6, 376 (2011)

11. M. Kong, J.L. Alvarado, W. Terrell Jr, C. Thies, Int J Heat Mass Tran 101, 901-914 (2016)

12. Z. Wu, L. Wang, B. Sunden, Appl Therm Eng 60, 266-274 (2013)

13. W.I.I.A. Aly, J. Heat Transfer 138, 102401 (2016)

14. A. Tohidi, S.M. Hosseinalipour, M. Shokrpur, A.S. Mujumdar. Appl Therm Eng 76, 185-195 (2016)

15. S. Mirfendereski, A. Abbassi, M. Saffar-awal. Adv Powder Technol 26, 1483-1494 (2015)

16. A. Alimoradi, F. Veysi, Int J Therm Sci 107, 196-208 (2016)

17. A. Sciacovelli, V. Verda, E. Sciubba, Renew Sust Energy Rev 43, 1167-1181 (2015)

18. A. Zachar, Int J Heat Mass Trans 53, 3928-3939 (2010)

19. J.C. Kurnia, A.P. Sasmito, T. Shamim, A.S. Mujumdar. Appl Therm Eng 102, 849-860 (2016)

20. W. Kays, M. Crawford, B. Weigand. Convective Heat and Mass Transport (McGraw Hill, Singapore, 2005)

21. H. Shokouhmand, M.R. Salimpour, Int Commun Heat Mass 34, 753-761 (2007) 
22. T.M. Liou. Exp Fluids 13, 332-338 (1992)

23. P. Naphon, J. Suwagrai. Int J Heat Mass Trans 50, 444-451 (2007) 